\title{
Krebsnachsorge: Viel Aufwand mit unklarem Nutzen?
}

\author{
Klaus Becker ${ }^{\mathrm{a} *}$ Wolfgang Dörr ${ }^{\mathrm{b} *}$ Ulrike Leiter-Stöppke ${ }^{\mathrm{c} *}$ \\ ${ }^{a}$ Onkologie Lerchenfeld, Onkologische Allianz Hamburg, \\ ${ }^{\mathrm{b}}$ Klinik und Poliklinik für Strahlentherapie und Radioonkologie, Medizinische Fakultät Carl Gustav Carus der Technischen Universität \\ Dresden, \\ c Zentrum für Dermato-Onkologie, Universitätshautklinik Tübingen, Deutschland.
}

\section{Nachsorge bei soliden Tumoren}

Neue Therapieansätze und Früherkennungsmaßnahmen haben dazu beigetragen, dass die 5-Jahres-Überlebensraten bei zahlreichen Tumorerkrankungen, vor allem beim Prostatakarzinom, Hodentumor, Rektumkarzinom, Morbus Hodgkin und Non-Hodgkin-Lymphomen, in den letzten Dekaden deutlich verbessert werden konnten $[1,2]$. Dies bedeutet zugleich, dass immer mehr Patienten, bei denen nach Abschluss der onkologischen Primärtherapie kein Tumor mehr nachweisbar ist und damit eine Heilung grundsätzlich möglich ist, eine Nachsorge benötigen.

$\mathrm{Da}$ die Primärtherapie immer intensiver und komplexer wird und bei adjuvanten Therapiekonzepten bis zu 5 Jahre dauern kann, steigt das Risiko von Langzeitnebenwirkungen wie Osteoporose (z.B. durch Aromatasehemmer beim hormonabhängigen Mammakarzinom) oder Polyneuropathien (z.B. durch Oxaliplatin beim kolorektalen Karzinom). Ziele der Nachsorge sind die Stärkung der körperlichen und psychosozialen Rehabilitation sowie die Früherkennung kurativ behandelbarer Tumorrezidive, wie bestimmter Lokalrezidive beim Mammakarzinom und solitärer Leber- und Lungenmetastasen beim kolorektalen Karzinom. Auch (therapiebedingte) Zweitmalignome sollen durch die Nachsorge möglichst so frühzeitig erkannt werden, dass sie kurativ behandelbar sind. Allein die Tatsache, dass die überlebenden Patienten älter werden, erhöht die Wahrscheinlichkeit, dass sie ein weiteres, altersabhängiges Zweitmalignom erleben. Durch sekundäre Leukämien gefährdet sind z.B. Patienten mit Hodentumoren, die mit Etoposid behandelt wurden, sowie Patientinnen mit Mammakarzinom, die Anthracycline erhielten. Nach einer Strahlentherapie des Prostatakarzinoms steigt

\footnotetext{
*Alle Autoren haben zu gleichen Teilen zu diesem Artikel beigetragen.
}

unter anderem das Risiko für Rektum- und Blasentumoren [3]. Patientinnen mit Mammakarzinom haben außerdem häufig ein erhöhtes Risiko für kolorektale Karzinome und Endometriumkarzinom.

Die Analyse der Daten von etwa 32500 Patienten mit Morbus Hodgkin aus 16 nordamerikanischen und europäischen Krebsregistern zeigte auch noch 25 Jahre nach der Diagnose ein erhöhtes Risiko für die Entwicklung sekundärer Malignome [4]. Überlebende von Malignomen im Kindesalter sind nicht nur durch Zweittumoren, sondern häufig auch durch multiple nachfolgende Tumoren gefährdet. Einer USamerikanischen Studie zufolge entwickelten von den mindestens 5 Jahre überlebenden Patienten $(\mathrm{n}=14$ 358) mit einem Zweitmalignom ( $\mathrm{n}=1382 ; 9,6 \%$ des Gesamtkollektivs) 386 $(27,9 \%)$ ein Tertiärmalignom und 153 sogar mehr als 2 Folgemalignome [5].

\section{Nachsorge am Beispiel Mammakarzinom, Ovarialkarzinom und Kolonkarzinom}

Vor dem Hintergrund der angestrebten Prognoseverbesserung hängt der Nutzen von Untersuchungen zur Früherkennung von Metastasen vor allem davon ab, ob eine Metastasierung überwiegend systemisch oder lokal erfolgt. Beim Mammakarzinom als vorwiegend systemisch metastasierender und dann nicht mehr kurativ behandelbarer Tumorerkrankung zählt bei der Nachsorge neben der körperlichen Untersuchung und Selbstuntersuchung der Brust nur die regelmäßige Mammographie zu den in Leitlinien empfohlenen Untersuchungen. In den Richtlinien der großen Fachgesellschaften (Deutsche Krebsgesellschaft, European Society of Medical Oncology, American Society of Clinical Oncology) nicht aufgeführt sind dagegen unter anderem die routinemäßige Bestimmung von Tumormarkern, Knochenszintigraphie, Röntgen-Thorax, Ultraschall der Leber und Positronen-Emissionstomographie (PET) [6].

\begin{tabular}{ll}
\hline KARGER & ( ) 2012 S. Karger GmbH, Freiburg \\
0378-584X/12/3514-0008\$38.00/0 \\
$\begin{array}{l}\text { Fax+497614520714 } \\
\text { Information@Karger.de } \\
\text { www.karger.com }\end{array}$ & $\begin{array}{l}\text { Accessible online at: } \\
\text { www.karger.com/onk }\end{array}$
\end{tabular}


Tab. 1. Beurteilung der Zuständigkeit für die Früherkennung eines Rezidivs im Rahmen der Nachsorge aus Sicht von Hausärzten und Onkologen (modifiziert nach [11])

\begin{tabular}{lcl}
\hline Zuständigkeit für die Früherkennung eines Rezidivs & Hausärzte, \% & Onkologen, \% \\
\hline Vollständig Onkologe & 1 & 54 \\
Hauptsächlich Onkologe & 2 & 44 \\
Teils Hausarzt, teils Onkologe & 30 & 2 \\
Hauptsächlich Hausarzt & 54 & 0 \\
Vollständig Hausarzt & 13 & 0 \\
\hline
\end{tabular}

Tab. 2. Beurteilung der Zuständigkeit für die Früherkennung eines Zweitmalignoms im Rahmen der Nachsorge aus Sicht von Hausärzten und Onkologen (modifiziert nach [11])

\begin{tabular}{lcc}
\hline Zuständigkeit für die Früherkennung eines Zweitmalignoms & Hausärzte, \% & Onkologen, \% \\
\hline Onkologe vollständig & 1 & 2 \\
Onkologe hauptsächlich & 1 & 22 \\
Teils Onkologe, teils Hausarzt & 2 & 32 \\
Hausarzt hauptsächlich & 35 & 29 \\
Hausarzt vollständig & 61 & 15 \\
\hline
\end{tabular}

Bei Patientinnen mit Ovarialkarzinom in vollständiger Remission nach platinhaltiger Erstlinien-Therapie waren in einer randomisierten Studie keine Vorteile einer frühen Chemotherapie (Therapiebeginn bei erhöhtem Cancer Antigen-125 (CA125)) im Vergleich zu einer späten Chemotherapie (Therapiebeginn bei klinischem oder symptomatischem Rezidiv) zu sehen. Bei einem medianen Überleben von 25,7 Monaten (frühe Intervention) bzw. 27,1 Monaten (späte Intervention) war die Prognose nicht unterschiedlich (Hazard Ratio (HR) $0,98 ; \mathrm{p}=0,85)$, dagegen war die Zeit bis zur Verschlechterung der Lebensqualität bei später eingeleiteter Chemotherapie signifikant länger (HR 0,71; $\mathrm{p}=0,002)$ [7]. Beim Ovarialkarzinom wird im Rahmen der Nachsorge daher keine Bestimmung von Tumormarkern empfohlen.

Beim Kolonkarzinom ist in der Nachsorge die Untersuchung auf Leber- und Lungenmetastasen prognostisch relevant, da aufgrund der hier häufig lokalen Metastasierung durch eine R0-Resektion mindestens 30\% (Lebermetastasen) bzw. mehr als 50\% (Lungenmetastasen) der Patienten trotz Fernmetastasierung geheilt werden können. Daher wird in der S3-Leitlinie der Deutschen Krebsgesellschaft die regelmäBige Bestimmung des carcinoembryonalen Antigens (CEA) und die regelmäßige Sonographie des Abdomens empfohlen [8]. Die Leitlinien der American Society of Clinical Oncology (ASCO) gehen sogar noch einen Schritt weiter: sie empfehlen - neben der Bestimmung des CEA - die Durchführung einer Abdomen- und Thorax-Computertomographie (CT) 1-mal pro Jahr für 3 Jahre nach der primären Diagnose. Diese Empfehlung gilt allerdings nur für Patienten, die fit genug sind für eine eventuelle Metastasenresektion, wobei der Nutzen einer CT des Thorax auch in den USA umstritten ist [9].

\section{Follow-up im Stadium IV}

In der metastasierten Situation stehen die Symptomlinderung und die Lebensverlängerung unter Berücksichtigung der Lebensqualität im Vordergrund. Regelmäßige Follow-up-Untersuchungen können dazu beitragen, frühzeitig eine Progression zu erkennen und zum optimalen Zeitpunkt eine therapeutisch sinnvolle Intervention einzuleiten. Zur Vorgehensweise (strukturiert vs. individuell; mit Bildgebung vs. klinisch) gibt es allerdings keine validen Daten, und auch die Leitlinien geben für diese Patientengruppen entweder keine Empfehlungen (Mammakarzinom, Kolonkarzinom) oder enthalten nur Angaben mit niedrigem Evidenzgrad (nichtkleinzelliges Lungenkarzinom (NSCLC)). Neben dem potenziellen Nutzen entsprechender Untersuchungen sollten in diesem Setting aber immer auch mögliche Belastungen des Patienten durch eine Überdiagnostik und Übertherapie sowie mögliche Risiken, beispielsweise durch Kontrastmittel-Zwischenfälle, berücksichtigt werden.

\section{Ökonomische Aspekte}

Bei den direkten Kosten für die Versorgung von Krebspatienten wird in den USA in den nächsten Jahren eine dramatische Kostensteigerung von 104 Milliarden US-\$ (2006) auf 173 Milliarden US-\$ (2020) erwartet [10]. Zurückgeführt wird dies sowohl auf die steigenden Therapiekosten als auch auf die zusätzlichen Aufwendungen für die Betreuung der Patienten. Vor diesem Hintergrund sollten bei der Nachsorge nur Tumormarker bestimmt und bildgebende Untersuchungen veranlasst werden, für die bei positivem Befund therapeutische Interventionen mit nachgewiesenem Benefit verfügbar sind.

\section{Zuständigkeiten}

Nach Abschluss der Primärbehandlung wird ein Teil der Patienten vom Onkologen betreut, in anderen Fällen übernimmt der Hausarzt die Versorgung. Einige Patienten sind sowohl beim Onkologen als auch beim Hausarzt in Behandlung, während andere keine Nachsorge in Anspruch nehmen. Den Ergebnissen einer US-amerikanischen Studie zufolge bestehen bei den befragten Hausärzten und Onkologen deutliche Diskrepanzen hinsichtlich ihrer Zuständigkeit für die Früherkennung von Rezidiven (Tab. 1). Bei der Früherkennung von Zweitmalignomen sehen dagegen beide Gruppen die Zuständigkeit eher beim Hausarzt (Tab. 2) [11]. 
Abb. 1. Arztbesuche (Millionen/Jahr) in verschiedenen Phasen der Tumorerkrankung, 2005-2020 (modifiziert nach [12]).

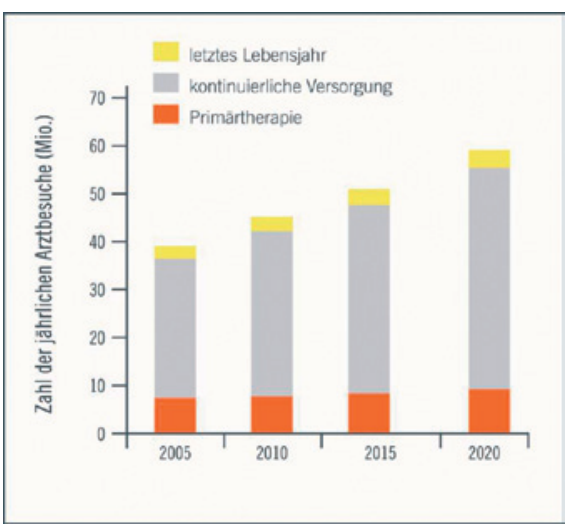

Abb. 2. Entdeckung des ersten Rezidivs (modifiziert nach [17]).

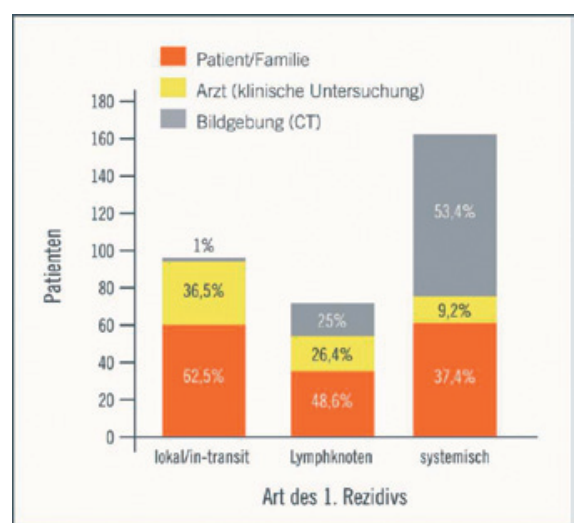

Tab. 3. Risiko eines 1 . Rezidivs beim kutanen Melanom in Abhängigkeit vom Tumorstadium (modifiziert nach [13])

\begin{tabular}{lllll}
\hline Jahre nach Diagnose & Stadium IA & Stadium IB & Stadium II & Stadium III \\
\hline 1 & $1: 152$ & $1: 37$ & $1: 7$ & $1: 3$ \\
2 & $1: 125$ & $1: 24$ & $1: 8$ & $1: 5$ \\
3 & $1: 167$ & $1: 40$ & $1: 13$ & $1: 10$ \\
4 & $1: 152$ & $1: 44$ & $1: 19$ & $1: 14$ \\
5 & $1: 167$ & $1: 58$ & $1: 23$ & $1: 14$ \\
6 & $1: 152$ & $1: 52$ & $1: 24$ & $1: 16$ \\
7 & $1: 200$ & $1: 71$ & $1: 29$ & $1: 14$ \\
8 & $1: 179$ & $1: 77$ & $1: 32$ & $1: 21$ \\
9 & $1: 217$ & $1: 72$ & $1: 46$ & $1: 30$ \\
\hline
\end{tabular}

\section{Ausblick}

Die in der Vergangenheit beobachtete Zunahme von Tumorpatienten, die kontinuierlich behandelt werden, dürfte sich auch zukünftig fortsetzen. Für die USA wird ein Anstieg der Tumorpatienten von 11,8 Millionen (2005) auf 18,2 Millionen (2020) angenommen. Die Zahl der jährlichen Arztbesuche dürfte damit von 38 Millionen auf 57 Millionen steigen, wobei die größte Zunahme bei den Patienten mit kontinuierlicher Versorgung erwartet wird (Abb. 1) [12].

\section{Nachsorge beim Melanom}

Ziele der Nachsorge beim malignen Melanom der Haut sind die Früherkennung von Rezidiven, die eine prognostisch günstige R0-Resektion ermöglichen, die Früherkennung von Zweitmelanomen, die Anleitung des Patienten zu protektiven Maßnahmen und zur Selbstuntersuchung, die Rückversicherung des Patienten und die Abklärung, ob der Patient von einer adjuvanten Therapie oder Teilnahme an einer klinischen Studie profitieren kann.

Da die Inzidenz des Melanoms in Deutschland in den letzten Jahrzehnten auf inzwischen 15-20 Fälle/100 000 Einwohner/Jahr deutlich zugenommen hat, ist auch der Bedarf an Nachsorgeuntersuchungen erheblich gestiegen. Zu berücksichtigen ist dabei allerdings, dass aufgrund der verbesserten Früherkennung mehr als 50\% der Fälle dünne Melanome mit einer Tumordicke $\leq 1,0 \mathrm{~mm}$ betreffen.

\section{Früherkennung von Rezidiven und Zweitmelanomen}

Die bei Melanomen im Stadium IA (T1a N0 M0; Tumordicke $<1,0 \mathrm{~mm}$; ohne Ulzeration) ermittelten sehr niedrigen Risikoraten (Tab. 3) [13] haben dazu geführt, dass die bisher gültigen Nachsorgeschemata bei dünnen Melanomen insgesamt $(\leq 1,0 \mathrm{~mm})$ infrage gestellt werden. Im Stadium IA könnte auf entsprechende Untersuchungen verzichtet werden, während im Stadium IB in den ersten 3 Jahren eine 2-mal jährliche Nachsorge empfohlen und danach eine Reduktion diskutiert wird. In den Stadien II und III werden aufgrund des hier sehr hohen Rezidivrisikos in den ersten 3 Jahren engmaschige Kontrollen mit bis zu 4 Untersuchungen pro Jahr empfohlen.

Den Einfluss der Rezidiv-Früherkennung auf die Prognose zeigen die Ergebnisse einer prospektiven Studie an der Hautklinik in Tübingen, in der die Daten von 1969 Patienten mit kutanem Melanom im Stadium I-III analysiert wurden. Im Nachsorgezeitraum von 1996 bis 1998 wurde bei 112 Patienten ein Rezidiv diagnostiziert, das auf Basis der Tumormasse und Operabilität einer frühen oder fortgeschrittenen Wachstumsphase zugeordnet wurde. Bei einer 10-Jahres-Überlebensrate von $35 \%$ im Gesamtkollektiv hatten Patienten mit einem früh erkannten Rezidiv $(n=59)$ eine signifikant bessere Prognose als Patienten mit einem später diagnostizierten Rezidiv (10-Jahres-Gesamtüberleben: $42,6 \%$ vs. $25,6 \%$; $\mathrm{p}=$ 0,013). Der Überlebensvorteil durch die Rezidiv-Früherkennung war auch unter Berücksichtigung des Lead Time Bias evident [14]. 
Patienten mit malignem Melanom haben ein Risiko von 3-12\% für die Entwicklung eines Zweitmelanoms [15], das bei einer standardisierten ärztlichen Nachsorge mit klinischer Untersuchung im Durchschnitt eine niedrigere Tumordicke (0,90 vs. $2,27 \mathrm{~mm} ; \mathrm{p}=0,008)$ aufweist als der Primärtumor [15] und die Prognose des Erstmelanoms damit nicht verschlechtert $[15,16]$. Patienten entdecken bei der Selbstuntersuchung dagegen eher dickere Zweitmelanome an gut sichtbaren Stellen [15].

\section{Sekundärprophylaxe und Anleitung zur Selbstuntersuchung}

Bei adäquater Schulung und Motivation sind Patienten in der Lage, Rezidive im Rahmen einer Selbstuntersuchung zu entdecken. In einer am Memorial Sloan-Kettering Cancer Center durchgeführten Studie bei Patienten mit einem Melanom im Stadium III, die später ein Rezidiv entwickelten, war dieses in 47\% der Fälle vom Patienten selbst oder von den Angehörigen entdeckt worden. Am häufigsten wurden bei der Selbstuntersuchung lokoregionäre Rezidive erkannt. Fernmetastasen wurden dagegen überwiegend vom Arzt bei der Nachsorge durch CT-Untersuchungen diagnostiziert (Abb. 2) [17].

\section{Kosten-Nutzen-Relation}

Die Kosten-Nutzen-Relation von Nachsorgeuntersuchungen variiert in Abhängigkeit vom Tumorstadium. In Anbetracht des im Stadium I sehr niedrigen Rezidivrisikos und der relativ häufigen Generierung falsch positiver Befunde, die weiter abgeklärt werden müssen, sind bei Einsatz bildgebender Verfahren die Aufwendungen zur Entdeckung eines Rezidivs im Stadium IA/B am höchsten. Da mehr als 50\% aller Untersuchungen bei Patienten mit niedrigem Rezidivrisiko durchgeführt werden, sollten zur Verbesserung der Kosten-Nutzen-Relation strukturierte stadien- und risikoadaptierte Nachsorgeschemata etabliert werden. Bei Patienten im Stadium I sollte auch der Verzicht bzw. die Restriktion bildgebender Verfahren diskutiert werden $[18,19]$.

\section{Follow-up im Stadium IV}

Für das Follow-up von Patienten mit metastasiertem Melanom machen die Leitlinien keine verbindlichen Angaben, sondern sehen ein individuelles Management vor. Nach R0Resektion von Fernmetastasen können klinische Untersuchungen und Bluttests (Laktat-Dehydrogenase (LDH) und S100) im Abstand von 6 Wochen erwogen werden. Für bildgebende Verfahren wird ein 3-monatiges Intervall diskutiert. Nach einem rezidivfreien Intervall von 6 Monaten können die Abstände der Staging-Untersuchungen verlängert werden.

\section{Nachsorgebedingte Strahlenexposition und daraus resultierendes Risiko für Zweitkarzinome}

Bei der Analyse eines möglichen Zweitkarzinom-Risikos infolge einer Strahlenexposition im Rahmen der Nachsorge
Abb. 3. Lebenszeitrisiko für solide $\mathrm{Tu}-$ moren in Abhängigkeit von der Strahlenexposition für Männer und Frauen in Abhängigkeit vom Alter (modifiziert nach [22]).

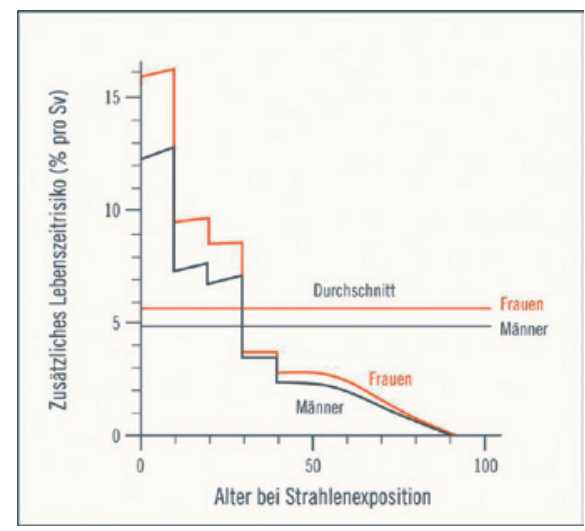

sind außer der Strahlendosis durch bildgebende Verfahren verschiedene weitere Risikofaktoren zu berücksichtigen.

Bei Patienten, deren Tumorerkrankung durch die Primärtherapie geheilt werden konnte, ist das Lebensalter der wichtigste Risikofaktor. Unabhängig von einer vorausgegangenen Therapie liegt die Wahrscheinlichkeit, in den folgenden 5 Jahren spontan an einem weiteren Tumor zu erkranken, bei Männern im Alter von 50, 60 und 70 Jahren in der Größenordnung von $1,5 \%, 5 \%$ und $10 \%$. Bei Frauen steigt die Risikorate von 2\% (50 Jahre) auf 5,4\% (70 Jahre). Diese Abschätzungen beruhen auf der Erfassung der Cancer Research UK [20]. Vergleichbare Ergebnisse lassen sich aus der Altersstatistik für Deutschland ableiten [21].

Weitere Risikofaktoren - unabhängig von der Art der Therapie - stellen eine genetische Prädisposition (z.B. Mammakarzinom), bestimmte virale Infektionen (z.B. Zervixkarzinom) oder die gleichzeitige Einwirkung von karzinogenen Faktoren auf verschiedene Organe (z.B. Tabakkonsum bei Kopf-Hals-Tumoren und Lungenkarzinom) dar [3]. Zudem müssen weitere Therapien (Chemotherapie, zielgerichtete Therapien) und ihre Interaktion mit der Strahleneinwirkung berücksichtigt werden, wofür allerdings nur in Ausnahmefällen verlässliche Daten vorliegen.

Nach Abschätzungen der International Commission on Radiological Protection (ICRP) beträgt das Lebenszeitrisiko für das Auftreten einer strahleninduzierten Krebserkrankung in der Gesamtbevölkerung etwa 5\%/Sv, wobei Kinder vor allem aufgrund der zum Zeitpunkt der Strahlenexposition längeren Lebenserwartung ein deutlich höheres Tumorrisiko haben als ältere Menschen; Frauen sind etwas empfindlicher als Männer (Abb. 3) [22].

Berechnungen auf Basis der Krebsmortalität von 1995 zeigen: Ausgehend von einem Lebenszeitrisiko für eine tödlich verlaufende Tumorerkrankung von 22,1\% (Frauen) und 26,4\% (Männer) erhöht beispielsweise die homogene Ganzkörper-Strahlenexposition mit $20 \mathrm{mSv}$ das Risiko bei einer Frau im Alter von 25 Jahren rechnerisch um 0,15\% auf 22,25\%; bei einem 60-jährigen Mann steigt das Risiko für eine tödlich verlaufende Krebserkrankung rechnerisch um 0,03\% auf $26,43 \%$. 
Im Rahmen der Strahlentherapie treten - im Gegensatz zur diagnostischen Strahlenexposition - sehr starke DosisInhomogenitäten auf, sodass derartige Abschätzungen auf Basis der ICRP-Werte bei einer therapeutischen Strahlenanwendung nur sehr bedingt anwendbar sind. Weiterhin ist $\mathrm{zu}$ berücksichtigen, dass bei von einem Tumor geheilten Patienten bereits das Ausgangsrisiko aufgrund der oben genannten Risikofaktoren möglicherweise erhöht ist, sodass die Erhöhung des Risikos durch die Strahlenexposition relativ abnehmen kann.

Als Beispiel für den Einfluss einer Strahlentherapie auf das Risiko von Zweitkarzinomen sei hier eine US-amerikanische Studie bei Prostatakarzinom-Patienten genannt, die anhand der Daten des Surveillance, Epidemiology, and End Results (SEER)-Krebsregisters durchgeführt wurde. Von etwa 17000 Patienten, die nach einer Strahlentherapie mindestens 5 Jahre überlebten, entwickelten 1185 einen Zweittumor. Unter Berücksichtigung der altersbedingt zunehmenden Grundtumorinzidenz dürften allerdings nur etwa 120-150 dieser Zweit- malignome, die sich hauptsächlich in Harnblase und Rektum sowie in der Lunge manifestierten, vorrangig auf die Strahlentherapie zurückzuführen sein [23].

Zusammenfassend ist festzustellen, dass das aus einer Strahlenexposition im Rahmen der initialen Strahlentherapie und vor allem der Tumornachsorge resultierende zusätzliche Risiko für eine Tumorerkrankung zumindest bei Erwachsenen sehr niedrig ist. Es wiegt den Nutzen der Therapie bezüglich der lokalen Tumorkontrolle und -heilung und der Diagnose von Rezidiven und Zweittumoren - vor allem in Anbetracht der langen Latenzzeiten bis zur Sekundärtumor-Manifestation - bei weitem nicht auf [3].

\section{Disclosure Statement}

Die Autoren waren Referenten beim «2. Interdisziplinären Expertenforum Hautkrebs», das von Bristol-Myers Squibb veranstaltet und unterstützt wurde.

\section{Literatur}

1 Kohler BA, Ward E, McCarthy BJ, et al.: Annual report to the nation on the status of cancer, 19752007, featuring tumors of the brain and other nervous system. J Natl Cancer Inst 2011;103:714-736.

$\checkmark 2$ Malvezzi M, Arfé A, Bertuccio P, et al.: European cancer mortality predictions for the year 2011. Ann Oncol 2011;22:947-956.

3 Dörr W, Herrmann T: Second tumors after oncologic treatment. Strahlenther Onkol 2008;184:67-72.

$\checkmark 4$ Dores GM, Metayer C, Curtis RE, et al.: Second malignant neoplasms among long-term survivors of Hodgkin's disease: a population-based evaluation over 25 years. J Clin Oncol 2002;20:3484-3494.

$\checkmark 5$ Armstrong GT, Liu W, Leisenring W, et al.: Occurrence of multiple subsequent neoplasms in longterm survivors of childhood cancer: a report from the childhood cancer survivor study. J Clin Oncol 2011;29:3056-3064.

6 Khatcheressian JL, Wolff AC, Smith TJ, et al.: American Society of Clinical Oncology 2006 update of the breast cancer follow-up and management guidelines in the adjuvant setting. J Clin Oncol 2006;24:5091-5097.

7 Rustin GJ, van der Burg ME, Griffin CL, et al.: Early versus delayed treatment of relapsed ovarian cancer (MRC OV05/EORTC 55955): a randomised trial. Lancet 2010;376:1155-1163.

${ }_{8}$ Schmiegel W, Reinacher-Schick A, Arnold D, et al.: S-3 Leitlinie 'Kolorektales Karzinom'. Z Gastroenterol 2008;46:1-73.
9 Desch CE, Benson AB 3rd, Somerfield MR, et al.: Colorectal cancer surveillance: 2005 update of an American Society of Clinical Oncology practice guideline. J Clin Oncol 2005;23:8512-8519.

10 Smith TJ, Hillner BE: Bending the cost curve in cancer care. N Engl J Med 2011;364:2060-2065.

11 Cheung WY, Neville BA, Cameron DB, et al.: Comparisons of patient and physician expectations for cancer survivorship care. J Clin Oncol 2009;27: 2489-2495.

12 Warren JL, Mariotto AB, Meekins A, et al.: Current and future utilization of services from medical oncologists. J Clin Oncol 2008;26:3242-3247.

13 Leiter U, Buettner PG, Eigentler TK, et al.: Hazard rates for recurrent and secondary cutaneous melanoma: An analysis of 33,384 patients in the German Central Malignant Melanoma Registry. J Am Acad Dermatol 2012;66:37-45.

14 Leiter U, Buettner PG, Eigentler TK, et al.: Is detection of melanoma metastasis during surveillance in an early phase of development associated with a survival benefit? Melanoma Res 2010;20:240-246.

15 Francken AB, Shaw HM, Thompson JE: Detection of second primary cutaneous melanomas. Eur J Surg 2008;34:587-592.

16 Brobeil A, Rapaport D, Wells K, et al.: Multiple primary melanomas: implications for screening and follow-up programs for melanoma. Ann Surg Oncol 1997;4:19-23.
17 Romano E, Scordo M, Dusza SW, et al.: Site and timing of first relapse in stage III melanoma patients: implications for follow-up guidelines. J Clin Oncol 2010;28:3042-3047.

18 Garbe C, Paul A, Kohler-Späth H, et al.: Prospective evaluation of a follow-up schedule in cutaneous melanoma patients: recommendations for an effective follow-up strategy. J Clin Oncol 2003;21: 520-529.

19 Livingstone E, Windemuth-Kieselbach C, Eigentler TK, et al.: A first prospective population-based analysis investigating the actual practice of melanoma diagnosis, treatment and follow-up. Eur J Cancer 2011:47:1977-1989.

20 http://info.cancerresearchuk.org/cancerstats/incidence/age/ [zuletzt abgerufen 11.02.2012].

21 Krebs in Deutschland 2005/2006, Häufigkeiten und Trends, ed 7. Berlin, Robert Koch-Institut (Hrsg), Gesellschaft der epidemiologischen Krebsregister in Deutschland e.V. (Hrsg), 2010, www.rki.de/krebs.

22 Hall EJ: Intensity-modulated radiation therapy, protons, and the risk of second cancers. Int J Radiat Oncol Biol Phys 2006;65:1-7.

23 Brenner DJ, Curtis RE, Hall EJ, et al.: Second malignancies in prostate carcinoma patients after radiotherapy compared with surgery. Cancer 2000;88: 398-406. 